\title{
Student knowledge integration in learning mechanical wave propagation
}

\author{
Li Xie $\odot,{ }^{1,2}$ Qiaoyi Liu, ${ }^{2}$ Hui Lu, ${ }^{3, \dagger}$ Qingyong Wang, ${ }^{4}$ Jing Han, ${ }^{2}$ \\ XiuMei Feng® ${ }^{5, *}$ and Lei Bao $\odot^{2, *}$ \\ ${ }^{1}$ School of Physics and Optoelectronic Engineering, Yangtze University, Jingzhou, Hubei 434023, China \\ ${ }^{2}$ Department of Physics, The Ohio State University, Columbus, Ohio 43210, USA \\ ${ }^{3}$ School of Petroleum Engineering, Yangtze University, Wuhan, Hubei 430100, China \\ ${ }^{4}$ School of Physics, Northeast Normal University, Changchun, Jilin 130024, China \\ ${ }^{5}$ College of Physical Science and Technology, Central China Normal University, \\ Wuhan, Hubei 430079, China
}

(Received 19 January 2021; accepted 1 September 2021; published 21 September 2021)

\begin{abstract}
A deep understanding of mechanical waves is crucial for students to succeed in studying many advanced physics topics. Studies in existing literature have revealed that students often have widespread difficulties and misconceptions on wave propagation. This research develops and applies a conceptual framework model to examine students' understanding of mechanical wave propagation from the knowledge integration perspective. Based on the existing literature and interview results, the conceptual framework model of wave propagation was developed and used to guide the development of a multiple-choice test that targets the assessment of knowledge integration in students' understanding of wave propagation. The assessment was given to first-year college students enrolled in a calculus-based introductory physics course at a medium-ranking Chinese university. The results suggested that after traditional instruction the majority of the students only achieved intermediate level knowledge integration with fragmented knowledge structures and lacked a deep conceptual understanding of wave propagation. The conceptual framework and assessment outcomes were then used to inform the development of new instruction, which made explicit emphasis on teaching the central idea of the conceptual framework and making connections to the central idea within students' knowledge structures. The effectiveness of the instruction was evaluated with the same pool of college students separated into an experimental group and a control group. Pre-post testing results suggested that the new instruction significantly improved students' performance on questions that require an integrated knowledge structure, indicating advancement in knowledge integration and deep understanding.
\end{abstract}

DOI: 10.1103/PhysRevPhysEducRes.17.020122

\section{INTRODUCTION}

Understanding and improving students' conceptual understanding is a fundamental goal in physics education over the past few decades [1-5]. However, a large number of students often fail to obtain a deep understanding of physics concepts after the traditional instruction, which also makes it difficult for students to apply their understanding to new situations during problem solving. A major

\footnotetext{
*Corresponding author.

bao.15@osu.edu

Corresponding author. luhui@yangtzeu.edu.cn

Corresponding author. xiumeifeng@mail.ccnu.edu.cn

Published by the American Physical Society under the terms of the Creative Commons Attribution 4.0 International license. Further distribution of this work must maintain attribution to the author(s) and the published article's title, journal citation, and DOI.
}

obstacle arises from their everyday experiences and preconceived notions, often incompatible with the normative scientific views, which can be difficult to overcome through traditional instruction [6,7]. This is due to the fact that traditional instruction often lends itself to rote memorization and its applications [8]. As a result, many students may perform well on textbook problems with familiar contexts, which they can solve relying on lower-level skills such as memorizing and pattern matching, but fail to solve the problems with new and unfamiliar settings, which requires the student to have an integrated knowledge structure and a deeper conceptual understanding [9-12].

In order to characterize student understanding, a myriad of theories have been established in the fields of science education and cognitive psychology. A few popular ones include the knowledge in pieces framework, resources model, conceptual change, and misconceptions, all of which were used to describe and analyze students' conceptual understanding and problem-solving strategies [13-21]. For example, the resources model focuses on the constructive 
process of student thinking, which is viewed as being composed of context-sensitive pieces of knowledge that are activated at the moment to construct understandings $[13,20,22]$. Meanwhile, the misconception and conceptual change frameworks emphasize the identification of common patterns of student reasoning and views and pedagogical interventions to change nonscientific reasoning and views towards scientific ones [15,16]. Although these models provide interpretations of student learning behaviors from different cognitive and behavioral perspectives, it can be generally agreed that novices' knowledge is structured differently from that of the experts. The differences in students' conceptual understanding can be modeled in terms of how their knowledge structures are constructed, activated, and linked, which serves as the basis of the knowledge integration framework $[11,21,23]$.

The knowledge integration model typically distinguishes students into several developmental levels such as novice, intermediate, and expert [24-28]. The novice students' understandings are often situated in familiar contexts that they encountered from personal experience and classroom learning, which often result in fragmented and disorganized knowledge structures. When solving problems, they often focus on surface features of the problems, and directly match these contextual features with memorized algorithms, equations, and examples [29-32]. For intermediate level students, many of them have developed partial connections in their knowledge structures; yet they still rely on memorization at times. During problem solving, these students can demonstrate improved understanding compared to the novices; however, they often fail to solve problems with unfamiliar physical scenarios due to the lack of organization in their knowledge structures, which limits their capability of transferring the partial links to the new contexts [33-35]. At the expert level, students are able to develop well-connected knowledge structures, which allow them to achieve a deep understanding of the concept and apply it across various contexts to solve problems in a wide range of settings.

Therefore, to promote deep learning, it is important to develop assessment and instruction specifically targeting knowledge integration. However, very few assessment instruments utilize the model of knowledge integration to measure students' conceptual understanding. Moreover, research has suggested that traditional instruction often leaves students with incoherent and fragmented knowledge structures relying on memorization $[11,24,36]$. To bridge this gap in the literature, the conceptual framework model has been developed in recent studies, which provides an operational method to explicitly represent students' knowledge structures developed in learning a specific topic. It serves as a tool to operationally guide assessment and instruction to target knowledge integration [25-27,37].

In a conceptual framework model, a central idea serves as an anchor point connecting the related knowledge components, including the contextual features and intermediate reasoning and processes. The use of the central idea and the links among different knowledge components are the two signifying features of knowledge structures that can distinguish experts and novices. The experts use the central idea as an anchor point to form an integrated, hierarchical knowledge structure, such that they can apply the central idea consistently to solve problems across a wide variety of contexts. On the other hand, the novices often bypass the central idea, and directly link the surface features to the task goals, so that they can only solve problems with familiar contexts.

The conceptual framework model can provide valuable utility in modeling student learning through different theoretical perspectives. For example, students' misconceptions of a concept can be represented as unique pathways connecting different contextual and conceptual elements of a conceptual framework, which can help researchers and teachers to visualize and understand how certain misconceptions are structured. Through such representations, misconceptions can also be easily viewed as activations of unique sets of conceptual resources in the resources perspective. For example, the central idea in a conceptual framework is the primary resource that experts always use but is usually underdeveloped among novices. Therefore, the conceptual framework model can aid the resources modeling with explicit representations of student thinking in terms of the actual resource pieces and the possible connections among the resources.

By clearly representing the different knowledge structures of experts and novices, the conceptual framework model can operationally guide the development of assessment instruments to probe different pathways within students' knowledge structures. The assessment results can then inform the design of new instructional interventions to help students build the needed connections within their knowledge structures and gain a deep conceptual understanding. In a number of recent studies, the conceptual framework model has been applied to several topics in physics, including light interference [25], force motion [26], and momentum [27]. In this study, students' understanding of wave propagation is examined using the conceptual framework model. The concepts in mechanical waves serve as a foundation for more advanced topics in optics, electromagnetism, and quantum mechanics, and thus are crucial to understanding both classical and modern physics.

Although wave phenomena are ubiquitous in daily life, the underpinning concept of wave is difficult to understand intuitively, and many students often display common misconceptions, even after instruction [38-41]. Prior to studying mechanical waves, students have learned to focus on the force and motion of particlelike objects; however, the propagation of a mechanical wave is the collective movement of a multiparticle system, defined as the 
medium, which is novel to most students and difficult for them to conceptualize. In teaching the wave behavior, many new concepts are introduced that go beyond the usual particle-based description, such as wavelength, period, frequency, and amplitude, which also have connections to concepts used to describe particle-like objects, such as velocity and energy. As a result, understanding the nature of waves and its distinction from particlelike objects can be challenging for students. In addition, students' understanding of waves can also be interfered by their prior knowledge of particle motion, which is evident in students' tendency of using the particle features to explain wave phenomena $[42,43]$. All these difficulties suggest that it is challenging for students to develop integrated knowledge structures and achieve a deep understanding in learning mechanical waves.

In this research, a conceptual framework model of mechanical wave propagation is developed and applied to guide assessment on students' levels of knowledge integration in learning mechanical waves. The conceptual framework model and assessment results are further applied to revise instruction that aims to help students develop more integrated knowledge structures and improve their conceptual understanding of wave propagation. Specifically, this paper is divided into three parts:

Part 1: the development of conceptual framework model of mechanical wave propagation and its validation based on qualitative analysis of students' conceptual understanding from the knowledge integration perspective.

Part 2: the development of a wave propagation test using the conceptual framework model, and its application to probe students' levels of knowledge integration towards understanding wave propagation.

Part 3: a controlled experiment to investigate the extent to which conceptual-framework-based instruction, which explicitly emphasizes the central idea and its connections to different knowledge components, can improve student knowledge integration and promote deep understanding in learning mechanical wave propagation.

\section{PART 1: DEVELOPMENT OF A CONCEPTUAL FRAMEWORK ON MECHANICAL WAVE PROPAGATION}

\section{A. Expert and novice understanding of mechanical wave propagation}

The first step to develop the conceptual framework of a concept is to identify the central idea based on experts' views [25-27]. In the case of mechanical wave propagation, the expert central idea is that a mechanical wave is the propagation of a local mechanical disturbance through a medium as a collective motion of the local interactions within the medium. In this research, only idealized mediums are considered, which have the properties of being linear, nondispersive, and elastic. The behavior of wave propagation is solely determined by the properties of the medium, and not the source [43]. The wave source initiates a disturbance such as an oscillation, which determines the frequency, period, and initial amplitude of the wave that is being propagated. The medium then responds to this disturbance through a sequence of interactions between adjacent parts of the medium allowing the disturbance to propagate, which forms the commonly perceived wave phenomenon. Therefore, the speed of the wave propagation is fundamentally different from the speed of the local disturbance (e.g., the oscillation speed of the particle inside the medium) and is a macroscopic collective phenomenon that depends on the medium's properties such as density, tension, and elasticity.

Conversely, as shown by numerous studies on students' understandings of wave propagation, novice students often hold very different views on how the wave propagates. According to these studies, when describing and explaining wave propagation phenomena, novice students often employ the "particle model" or the "particle pulses model," where they follow the belief that a wave travels as a particlelike object and make analogies between the motion of a particle and the motion of a wave [38,43-47]. These naïve understandings appear to be inconsistent with the experts' central idea about wave propagation and are often difficult to change through traditional instruction. Even after instruction, many students were still confused between particle motion and wave propagation. They tend to use concepts of particle motion, such as force, energy, or particle-based kinematics, to describe wave propagation.

For more advanced students, their physics conceptual resources are better developed with more extensive links established among their knowledge elements. As a result, these students start to demonstrate reasoning with aspects of the expert model; however, the lack of a good understanding of the expert central idea often leads them to use equations without deeper understanding and reasoning. For example, many students have been found to heavily depend on using the equation $v=\lambda f$ to solve problems of wave speed. However, their reasoning was often incorrect, considering the wave speed as being proportional to the frequency of the wave without recognizing that frequency is the property of the source while wavelength and propagation speed are properties of the medium [48,49]. Meanwhile, widespread inconsistencies have also been observed among these students' understandings such that they often use inconsistent mixed ideas when solving questions of the same concept but with different context settings [41], a result that is also evident in students' learning of other concepts $[21,34,50,51]$. This inconsistency or mixing is a clear indication that these students' knowledge structures are fragmented in the forms of partially connected local links such that different inconsistent local links may be activated by different contexts 
and used in problem solving. It is not until students achieve the expert level when they start to develop an integrated knowledge structure that is fully connected with the expert central idea. Students at this level are then capable of applying the expert central idea across a wide range of familiar and novel contexts in problem solving, demonstrating a consistent expert-like deep understanding of the concept.

To summarize, the existing literature has documented rich information on students' common naïve conceptions and their ways of applying these different conceptions in problem solving. However, existing studies have documented mostly individual conceptions and learning behaviors without a comprehensive framework that can tie the various types of students' understandings into a coherent progression of knowledge integration. In the next section, a conceptual framework is developed that can model student learning with a more holistic account of students' reasoning in learning mechanical waves.

\section{B. The conceptual framework of wave propagation}

Based on the existing literature, it is evident that experts and novices have distinct understandings of wave propagation (i.e., wave model vs particle model), which they follow consistently. Meanwhile, students in the intermediate level often switch between the two main ideas during problem solving. As a result, in order to more completely represent the two different knowledge structures between novices and experts, a dual-structure conceptual framework is developed for wave propagation, in which the experts' and novices' models are laid out in parallel, each with its own central idea and connections. The full conceptual framework for wave propagation is shown in Fig. 1.

As represented in the conceptual framework, wave phenomena can be understood through two sets of knowledge structures. The section on the left of the conceptual framework is the experts' knowledge structure, in which the knowledge elements (e.g., contextual features, variables, and relations) are fully connected by different pathways through the expert central idea. The expert knowledge structure is organized hierarchically from its central idea to intermediate processes and to contextual features with fully connected links. The expert central idea clearly distinguishes the propagation of the wave from its source, which leads to two separate conceptual pathways that form the intermediate layers of reasoning processes: one for processing the source-determined properties (e.g., frequency, amplitude, etc.), and the other for the medium- determined properties (e.g., wavelength, speed, etc.). The reasoning processes also include mathematical and logical manipulations that can operationally process the relationships among the properties and contextual features and variables (see Fig. 1).

This fully connected knowledge structure allows the experts to reason from any given point of a contextual or conceptual component to reach the central idea and back to the problem settings with a flexible and comprehensive network of thinking pathways, which are represented with solid arrows in the conceptual framework. In learning and problem solving, the activation of a single element within the experts' knowledge structure can readily activate the entire web of knowledge hierarchy, giving experts a deep

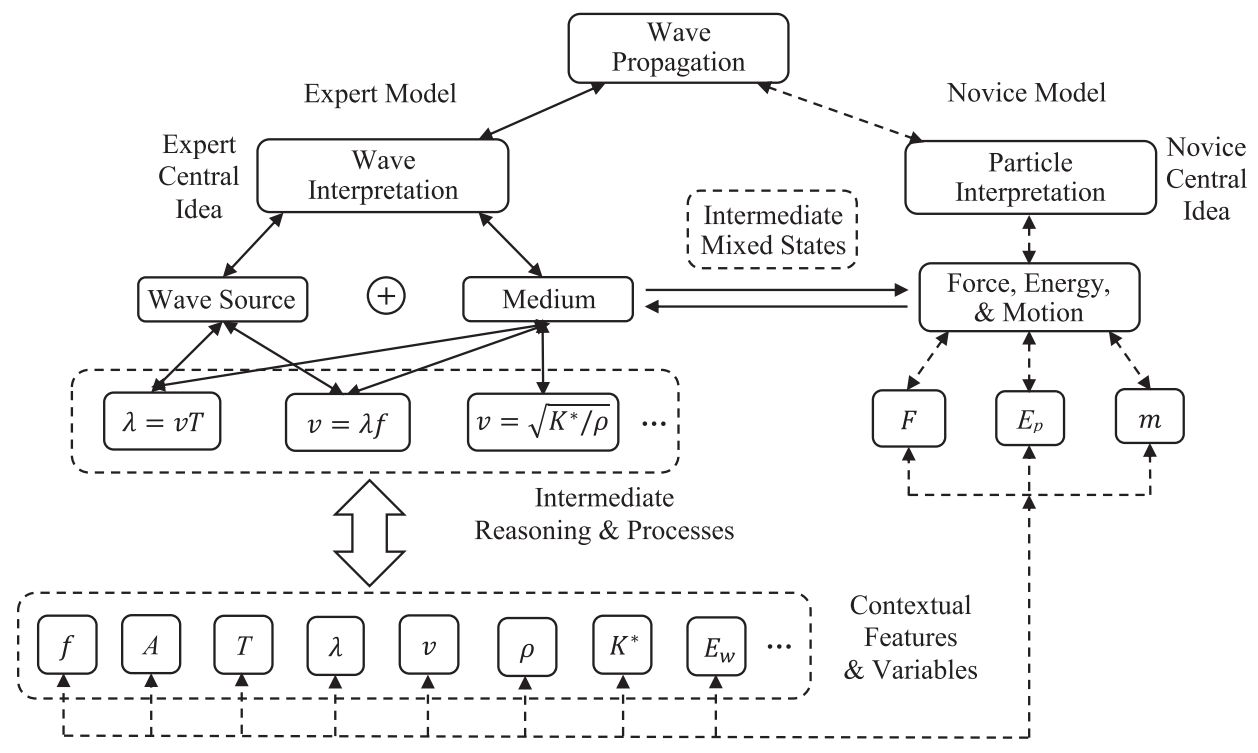

FIG. 1. The conceptual framework of wave propagation. Solid lines represent experts' conceptual pathways, while the dashed lines represent the links that novices often made. The wave interpretation represents the understanding that the propagation of a mechanical wave is determined by the medium. The particle interpretation treats the propagation of a wave as the motion of a particlelike object. Among the contextual features and variables, $K^{*}$ is the elasticity of the medium or the tension of the string, and $E_{w}$ is the energy of the wave, including the kinetic energy of the medium's motion and the potential energy of the medium's elastic deformation. 
and profound perspective to analyze different context configurations and transfer fluently from familiar situations to novel conditions.

Also shown in Fig. 1, the section on the right of the conceptual framework represents the novices' knowledge structure, which interprets waves as particlelike objects. Specifically, the propagation of a wave is understood in terms of the force, energy, and motion of the kinematic behaviors of a particle, which is referred to as the FEM particle model. In this model, students often associate the force $(F)$ involved in the source of a wave, such as the force exerted by a hand or a loudspeaker, as a factor influencing the motion of the wave $[43,52]$. The energy $(E)$ concept is a component in both the expert and novice models. While the experts typically do not associate energy with the speed of a wave, novices often interpret energy as a particle feature strongly associated with an applied force and/or the speed of wave motion $[22,45]$. To distinguish the difference, in the novices' knowledge structure, $E_{p}$ is used in the conceptual framework to represent the particle-based energy concept. For novice students, the motion $(M)$ of a wave is also commonly interpreted in terms of their knowledge of particle kinematics, which is again related to the force applied on a particle and the energy of the particle. Therefore, this FEM model includes a combination of heavily entangled intuitive understandings for describing particle-based behaviors.

As shown by research, students in the intermediate level have developed some fragments of the expert model, but they often switch back and forth between expert and novice models during problem solving [41]. Such inconsistency and transitions among students' behaviors indicate that students' knowledge structures are not static but are dynamically evolving and growing, and different contexts can activate different model elements and links between them, leading to the different reasoning pathways [21,53]. These are represented as intermediate mixed states in the conceptual framework to signify the mixing and switching between the expert and novice models.

It is also noted that the conceptual framework of wave propagation developed in this research is constructed differently from those developed in previous research [25-27]. In previous studies, students' naïve concepts are structured as isolated fragments with local connections without forming a complete knowledge structure. However, for the topic of wave propagation, there appears to exist a clear novice model that is based on a completely different set of central idea and reasoning processes, i.e., the FEM combination of concepts and the underpinning central idea of the particle-based interpretations of waves. Therefore, the conceptual framework of wave propagation is established with a dual-structure configuration that explicitly separates the expert and the novice models, which can more clearly represent the many forms of experts' and novices' conceptual pathways as well as the intermediate mixed states of knowledge development. This new dual-structure approach also extends the current constructs of the conceptual framework to allow more precise and versatile representations of students' knowledge structures and reasoning processes.

\section{Modeling student understanding using the conceptual framework}

In the existing studies on student learning of wave propagation [38-41], it is well established that students often hold many alternative concepts containing different conceptual elements and links, which form a general progression of developmental levels in knowledge integration. To make connections to the existing findings, student interviews were conducted to reexamine students' conceptions from a knowledge integration perspective. The interview results also provide qualitative evidence for validation of the conceptual framework model and demonstrate the utility of using the conceptual framework in modeling student learning behaviors.

The interviews were conducted with two open-ended questions on wave speed in different mediums (see Fig. 2), which were modified based on similar questions in the Mechanical Waves Conceptual Survey [41]. A total of 15 college freshmen from a calculus-based introductory physics course at a medium-ranking Chinese university were solicited to participate in the interviews. During the interviews, the students were asked to answer the questions in a think-aloud mode to describe in detail their reasoning processes. Each interview lasted approximately $20 \mathrm{~min}$, and students were each given a gift card for their participation in the interviews.

Using the conceptual framework of wave propagation, results from existing studies and interviews conducted in this research are summarized into three levels of knowledge integration described below. These levels are defined based on different developmental stages of student learning in the process of changing from the novice model to the expert model, which is aligned with the goal of instruction.

\section{The novice level}

Students in this level used the novice model consistently when answering all questions with different contexts. These students consistently described wave propagation using the particle model and connected the contextual

\footnotetext{
Question 1: Two students sing equally loudly. Student $X$ sings at a high pitch, while student Y sings at a lower pitch. Who will hear the other student's sound first? Why? Explain your reasoning.

Question 2: A girl holds a long, stretched

string and quickly moves her hand up and

down, creating a pulse that moves toward the

wall to which the string is attached. The

pulse reaches the wall in time $t_{0}$ (see figure).

What could she do to decrease the amount of

time it takes for the pulse to reach the wall?

Explain your reasoning.

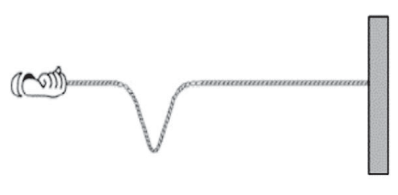

FIG. 2. Interview questions used in part 1 of this research. 
features and variables with the FEM interpretation through the novice central idea. Although some of the students gave the correct answers in limited situations, their explanations during the interviews demonstrated a lack of basic understanding of the role of the medium played in wave propagation, which is evident from the following interview excerpts:

Student A: (Response to question 1) "I think student $Y$ will hear the sound first because the pitch of the student $X$ is higher. A higher pitch means a higher frequency, so the sound emitted by student $X$ has higher energy making his sound travel faster." (Response to question 2) "you know, if you shake the string up and down more quickly, the string will gain more energy and higher frequency, causing faster speed of the pulse."

Student B: (Response to question 1) "Two students sing equally loudly, so their voices have the same energy, and they hear each other's sound at the same time." (Response to question 2) "the person should shake her hand harder to create a larger and wider pulse, so the pulse will move faster."

The two students, like most typical novices, used the FEM concept to describe how they would change the speed of wave propagation by altering the source conditions, and could not distinguish the energy of the wave from the energy of the oscillation at the source. For example, regardless of the medium, student A always linked frequency to force and energy. More specifically, a higher frequency was linked to more energy of the sound or the string pulse, which was further connected to a higher speed of the wave. In addition, student B relied on the amplitude (volume) of the wave to determine the wave's speed, which led to the correct answer in question 1 but demonstrated incorrect reasoning. In question 2 , the same student demonstrated incorrect reasoning between the force at the source and the wavelength, leading to the conclusion that a greater force could create a larger and wider pulse, which was believed to travel faster.

The misconceptions demonstrated by these two students are well documented in the literature $[39,43,45]$. Through the conceptual framework model, these students' behaviors can be interpreted as thinking pathways that directly connect the surface features to the FEM particle model shown in the right section of Fig. 1. These naïve thinking pathways form the novices' knowledge structures, which are centered around the FEM particle model.

\section{The intermediate level}

Students in the intermediate level have started to gain some basic understanding of the expert model, but the links they have established between the layers of knowledge structure were only locally connected, making them harder to be transferred to solve problems in new contexts. The students may develop mixed use of different models that incorporates pieces of both the expert model and the novice model $[34,35]$. For instance, student $\mathrm{C}$ memorized the equation of the speed of wave but with little conceptual understanding. The student then could only use a patternmatching strategy to match the equation with contextual features in the two questions.

Student C: (Response to question 1) "I remember the equation $v=\lambda f$. Student $X$ sings at a high pitch, em... the frequency of his voice is higher, the speed is faster. So, Student Y will hear the sound first." (Response to question 2) "I still prefer to use the equation $v=\lambda f$. If you want the pulse to move faster, you should increase the frequency by flicking the string more quickly."

Although this student's knowledge structure contained the second layer components of the expert model, they were in the form of memorized equations with little understanding connecting to the expert central idea, which resulted in the incorrect application of the equations. Meanwhile, many elements of the novice model still remained in this student's knowledge structure.

In addition, a number of students were found using different models inconsistently in their reasoning. Parts of both novice and expert models were activated by different contexts, and students switched back and forth in using these models in problem solving. The following two students' responses indicate this mixed use of models:

Student D: (Response to question 1) "the wave speed is related to the medium, and the speed of sound in air is about $340 \mathrm{~m} / \mathrm{s}$. Here, the medium is air, so two students will hear each other's voice at the same time." (Response to question 2) "To decrease time, we need to increase the speed of wave propagation. In this question, the girl should shake her hands harder...the greater force is put into the string to make the pulse go faster."

As revealed in the above excerpt, student D was able to apply some level of expert-like reasoning to obtain the correct answer in a familiar context (wave propagation in air) but reverted to the novice model using the FEM concept as the basis of reasoning, with little awareness of the contradiction, when facing an unfamiliar context (wave propagation in a string).

There were also students who recognized the conflicting ideas between novice and expert models, but it was difficult for them to arrive at a coherent conclusion. Usually, these students were left in an uncertain state due to not understanding the expert central idea. The following interview excerpts demonstrate this phenomenon:

Student E: (Response to question 1) "I would think student $Y$ would hear the sound first, but I remember the sound may be related to the air... but I think the higher pitch given in the question will affect the sound speed. I am not sure."

Student F: (Response to question 2) "If you can shake your hand harder or faster, the pulse will reach the wall in a shorter time. Oh...I have learned that the wave speed depends on the medium, but I feel that the more force you apply, the faster the object should move. I am not sure." 
The responses of the intermediate level students indicate that their knowledge structures have started to incorporate some elements from the expert model, yet components of the novice model were still frequently activated and caused confusion in students' reasoning. Being able to recognize the inconsistency between the two sets of ideas in reasoning is an encouraging sign of students attempting to connect multiple local links to form more integrated structures. However, due to the lack of deep understanding of the expert central idea, students at this level were unable to coordinate the components of different models to develop integrated knowledge structures. As a result, these students still held coexisting expert and novice types of understandings in partially fragmented knowledge structures, which often led to inconsistent activations of the different types of knowledge elements in problem solving [20,21].

\section{The expert level}

Students in the expert level were able to apply the expert model consistently in questions with a wide variety of contexts. Regardless of what medium a wave travels through, these students recognized that the speed of the wave is only related to properties of the medium, such as the tension of the string and the density of the medium. They also recognized that elements of the medium itself are not moving with the wave as the wave propagates. Below are two examples of the interview responses from two students with the expert model:

Student G: (Response to question 1) "the speed of the wave is only related to the medium, the information in this question such as equal loudness, lower pitch or higher pitch, is the distractor. The medium here is air, which determines the speed, so the two students should hear each other's sound at the same time." (Response to question 2) "the medium in this question is the string, and I know the wave speed is dependent on the tension and the density of the string. If the string is the same, we can increase the tension of the string to let the pulse go faster."

Student H: (Response to question 1) "The speed of sound is determined by the air and is independent of frequency, amplitude, and wavelength, so the sound speeds by these two students are the same. By the way, using the equation $v=\lambda f$ here is not correct." (Response to question 2) "If you want to increase the speed of wave propagation, you must change the nature of the medium. We can pull the string tighter with the same string. Only an alteration in the properties of the medium will cause a change in the speed."

From the interviews, it can be seen that these expert level students had a good understanding of the expert central idea and were able to apply it consistently in different contexts, indicating that they had developed fully integrated knowledge structures. Contextual factors, such as the sound pitch and the hand motion, did not affect their responses to questions related to wave propagation. Whether in a familiar context (wave propagation in the air) or an unfamiliar context (wave propagation in a string), these students could reason coherently using the expert central idea to arrive at the correct answers.

According to the interview results, 2 out of the 15 students were identified as in the novice level. They applied the novice central idea consistently to describe wave propagation, directly linking the contextual variables and features to the particle-based FEM model. Their reasoning pathways were linking through the novice model side of the conceptual framework in all contexts. Meanwhile, the majority of the interviewed students (11 out of 15) were found in the intermediate level. Most of these students used a mixture of different types of models in their reasoning. This mixed state implies that a significant fraction of these students had fragmented knowledge structures, with locally connected elements and links from both the novice and expert models. As a result, different contexts may cue different conceptual resources or model elements within their knowledge structures, leading to the mixed state in their reasoning. Finally, 2 students were identified as in the expert level. They applied the expert central idea consistently in different contexts to explain wave phenomena, indicating that they had developed integrated knowledge structures, with strong and global links between the expert central idea and other components of the conceptual framework.

As can be seen from the analysis, students at different levels of knowledge integration demonstrate unique types of thinking pathways that can be mapped in the conceptual framework, making it a useful tool to represent different mental states and knowledge structures. With the conceptual framework of wave propagation established, in the next part of research, the conceptual framework is applied to guide the development of a multiple-choice assessment to probe students' knowledge structures and evaluate their levels of knowledge integration.

\section{PART 2: ASSESSMENT OF KNOWLEDGE INTEGRATION IN MECHANICAL WAVE PROPAGATION}

\section{A. Design of the wave propagation test}

In the literature, there are several existing conceptual surveys on wave propagation [39-41,43,54]. However, these surveys were not designed to evaluate the levels of students' knowledge integration. Hence, this research will extend the current assessments to target students' knowledge integration in their learning of mechanical wave propagation.

Based on the conceptual framework of wave propagation established in part 1 of this research, a wave propagation test containing 15 multiple-choice questions was developed to investigate students' conceptual understanding and levels of knowledge integration. Among the 15 questions, Q1-Q8 were adapted from previous research on wave 
propagation $[41,43,46,52]$. The remaining questions were designed based on ones commonly used in instruction. The complete test is included in the Supplemental Material [55].

The assessment design uses a mixture of typical and atypical questions, which is a strategy shown effective in designing conceptual-framework-based assessment [2527]. Typical questions contain contextual settings that the students often encounter in lectures, textbooks, and homework, which can be solved with memorized solutions and problem-solving strategies. Meanwhile, atypical questions are designed with unfamiliar contexts that require the use of the expert central idea to solve correctly.

To specifically target students' levels of knowledge integration, features of the knowledge integration rubric developed by Linn et al. [11,23,24,56] are also used to guide the assessment design. The original knowledge integration rubric contains 6 levels of connectedness within students' knowledge structures, which include no answer, no link, partial link, full link, complex link, and systemic link. Among the different levels, no link indicates nonnormative ideas or links are elicited; partial link means that some normative ideas are elicited but with inconsistent partial connections; full link (and complex link) indicates that multiple normative ideas are elicited and connected with single (multiple) link(s) among them; systemic link represents extended connections to other normative ideas in related knowledge domains. These levels also match well with the taxonomy of structure of the observed learning outcomes (SOLO) [57], which models a student's knowledge structure in 5 progression levels of complexity of connections including prestructural for not understanding any related aspects, unistructural for focusing on one relevant aspect, multistructural for understanding several relevant aspects without connections in between, relational for being able to integrate different aspects into a coherent whole body of connected understandings, and extended abstract for being able to conceptualize at a higher level of abstraction and generalize to a new topic or area.

Synthesizing the existing assessment methods of knowledge integration discussed above, the 15 questions in the wave propagation test were designed into 3 question sets with differing degrees of knowledge connectedness (link type) and contextual saliency. These include single-link typical question set, multilink typical question set, and integrated-link atypical question set. The question design combines the existing knowledge integration rubric with the conceptual framework structure to probe the knowledge integration levels discussed in part 1.

The single-link typical set contains 5 typical questions (Q1, Q2, Q10, Q12, and Q14) that require single-link type of knowledge integration to answer correctly. These questions can be solved using a memorized statement of relation or an equation so that the indicated reasoning pathways are direct local links between the memorized elements and the task outcomes. For Q1, Q2, and Q10, the key statement is "sound can travel through different mediums (gas, liquid, and solid)" or "the sound speed is about $340 \mathrm{~m} / \mathrm{s}$ in air", which can be directly applied to reach the conclusions. For Q12 and Q14, the equation of $v=\lambda f$ or $v=\lambda / T$ is often applied, which most students memorize and can perform the routine "plug and chug." Based on the literature, teaching experiences, and student interviews, student behaviors in responding to these questions can be inferred. Typically, novice students would tend to use their particlebased FEM concept and obtain incorrect answers, but they might have occasional success based on memorization of similar outcomes. Meanwhile, expert and intermediate level students should be able to successfully answer most of these questions.

The multilink typical question set contains more complex typical questions that are designed with multiple conceptual components. This set includes 4 typical questions: Q9, Q11, Q13, and Q15, designed with contexts that are commonly seen in lectures and textbooks. Compared to the single-link questions, the multilink questions require that multiple local links related to both the wave source and the medium are activated and applied correctly at the same time in order to solve the questions. Although these questions are more complicated than those in the single-link set, they can still be answered using routine problem-solving procedures, such as pattern matching with isomorphic familiar problems and using memorized equations. Among these questions, Q9 and Q13 use a familiar context of the sound wave. In Q9, two sound waves are emitted from the same source and travel in different mediums (air and water), while in Q13, sound waves produced by two different sources travel in the same medium. In both questions, students are asked to compare the wavelengths, speeds, and frequencies of the waves. Q11 and Q15 ask the students to first calculate the period or the wavelength, and then use the equation $v=\lambda f$ to obtain the result. These questions all require multiple steps to solve correctly. In responding to these questions, it is expected that novice students would usually fail to obtain correct answers. Meanwhile, the intermediate level students should often be able to correctly answer a large fraction of these questions, and the expert level students should be able to correctly answer most of these questions.

The integrated-link atypical set includes 6 atypical questions designed with unfamiliar contexts to examine if students can successfully apply the expert central idea in novel situations, which indicates achieving an integrated knowledge structure. The integrated-link set includes 6 atypical questions, Q3-Q8, which are adapted and modified from the questions used in previous studies $[41,43,46,52]$. To answer these questions correctly, students need to understand the expert central idea and be able to successfully apply it in unfamiliar contexts, which include situations such as changing speed in a string and determining the motion of a particle in the medium. It is 
TABLE I. Question designs for assessment of knowledge integration levels.

\begin{tabular}{llcr}
\hline \hline Knowledge integration & Single-link typical & Multilink typical & Integrated-link atypical \\
& (Q1, Q2, Q10, Q12, Q14) & $(\mathrm{Q} 9, \mathrm{Q} 11, \mathrm{Q} 13, \mathrm{Q} 15)$ & $(\mathrm{Q} 3, \mathrm{Q} 4, \mathrm{Q} 5, \mathrm{Q} 6, \mathrm{Q} 7, \mathrm{Q} 8)$ \\
Novice level & Occasionally successful & Rarely successful & Guessing or using novice model \\
Intermediate level & Mostly successful & Often successful & Sometimes successful \\
Expert level & Always successful & Always successful & Mostly successful \\
\hline \hline
\end{tabular}

expected that in responding to these questions novice students would usually rely on guessing since the context and required knowledge elements would appear foreign to most of these students. Novice students might also use their naïve models when certain contextual features are in line with such models. Meanwhile, intermediate level students would also have difficulty with the atypical questions but might have success with some of them. For expert level students, being able to apply the expert central idea should allow them to successfully solve most of the atypical questions.

Designed with a mixture of differing degrees of knowledge connectedness and contextual saliency, the wave propagation test is expected to measure and identify students' knowledge integration levels. The expected (predicted) problem-solving behaviors from students at different knowledge integration levels are summarized in Table I.

\section{B. Research procedure}

The students involved in this research were first-year college students from a large-scale comprehensive university with a national ranking around 200 in China. A total of 312 students, who were enrolled in 6 classes of a calculusbased introductory physics course, took the wave propagation test at the beginning of the spring semester in 2020 before instruction on related topics in their college physics course. All the students had previously learned the relevant content of mechanical waves in their high school physics courses. Therefore, this test can be considered as a pretest to college physics as well as a delayed post-test of high school physics. In the following discussion, this test will be referred to as the pretest for comparison with a post-test conducted with 229 students from the same pool of students after their college physics instruction on mechanical waves. These students were given an hour to complete the test. It is also noted that the 15 students interviewed in part 1 of this research were selected from the same course in the previous academic year. These students did not take any pre-post tests and were not involved in any instructional interventions.

The main purpose of this part of research is to evaluate students' conceptual understanding in terms of their levels of knowledge integration by using the wave propagation test designed based on the conceptual framework established in part 1 of this research. Statistical significances in comparing results of different question sets were determined using a one-way ANOVA and further explored using $t$ tests and Cohen's $d$ effect size.

\section{Quantitative study on students' knowledge structure}

Students' pretest scores on the three question sets are listed in Table II. For the pretest, the Cronbach's $\alpha$ is 0.699, indicating sufficient reliability achieved $(>0.65)$ [58]. The assessment outcomes are consistent with the expectations shown in Table I, with the single link set having the highest score and the integrated-link set having the lowest score. A one-way ANOVA shows significant differences between the three question sets $[F(2,933)=25.615, p<0.001]$, which are more clearly demonstrated with pairwise $t$ tests between different question sets $\left[t_{(S M)}(311)=23.79, p<0.001, d=\right.$ $1.50 ; t_{(M I)}(311)=15.05, p<0.001, d=1.04 ; t_{(S I)}(311)=$ 38.34, $p<0.001, d=2.75]$. The results demonstrate that the question design shown in Table I can discriminate students' scores with a high level of statistical significance.

To examine how students at different overall performance levels may respond to the three question sets, the score distributions for the different question sets are plotted in Fig. 3. A histogram of total score frequency is displayed in the background to show the distribution of students across the different overall performances. The curves shown are based on data from both pretest and post-test combined to provide a larger number of samples in each performance level, especially at the higher performing levels.

As shown in Fig. 3, scores on the three question sets are similarly low for all students with low total scores $(<30 \%)$. Referencing to the expected students' performances listed in Table I, a total score below $30 \%$ can be used to indicate a novice level of understanding that leads to poor performances on all types of questions except for occasional success on the single-link ones. As the total score increases, performance gaps between the different question sets are more pronounced, suggesting that students in this range have started to perform well on simple and more complex typical questions using memorization but without establishing a deep understanding. As the total score further improves, the single-link typical question performance

TABLE II. Students' pretest scores on the three question sets. The scores shown are in percentage scale.

\begin{tabular}{llcc}
\hline \hline Question set & Question type & Mean $(N=312)$ & SE \\
\hline Single-link & Typical question & 65.64 & 1.77 \\
Multilink & Typical question & 39.90 & 1.67 \\
Integrated-link & Atypical question & 23.61 & 1.45 \\
\hline \hline
\end{tabular}




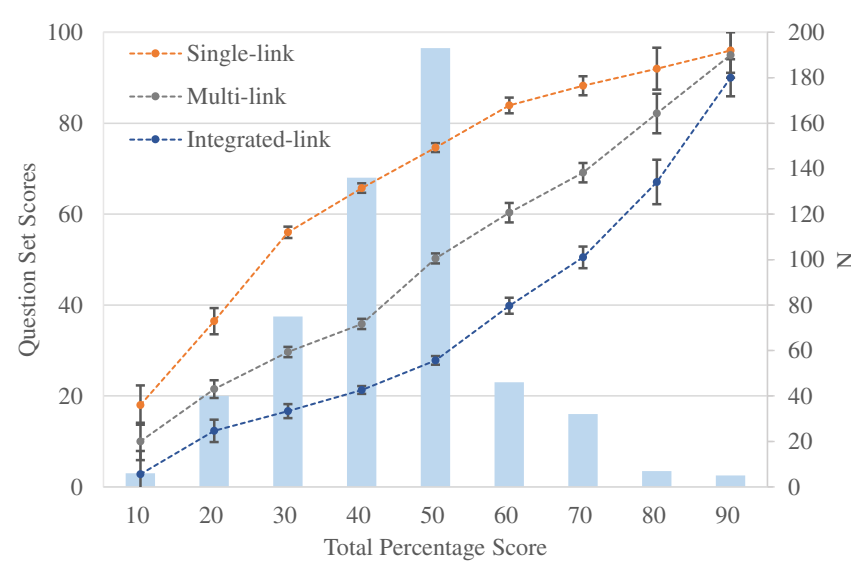

FIG. 3. Plot of question set subscores across total scores (with error bars denoting standard error) for all pre- and post-test data combined. Frequency of total score distribution is shown as a bar chart in the background, with absolute count of students falling into each range shown on the right axis.

quickly reaches the mastery level and students' performance on multilink typical questions starts to show significant improvement. Performance on integrated-link atypical questions also starts to catch up. The results indicate that students have developed partially integrated knowledge structures that allow them to apply their understanding in some unfamiliar situations. Finally, students with the highest scores $(>80 \%)$ show a minor difference between their scores on typical and atypical questions, indicating that they have achieved a deep understanding with a well-integrated knowledge structure (also see Table I). These patterns of scores on different questions reveal a general progression of student knowledge integration that matches well with the novice, intermediate, and expert levels discussed in part 1 and summarized in Table I.

Synthesizing the assessment data with the qualitative results discussed in part 1 , students at the three levels of knowledge integration are expected to have different performances on questions with different designs of link structure and context saliency. Based on the performance gaps among the differently designed questions shown in Table II and Fig. 3, mean total score of the test appears to be a useful indicator for the different knowledge integration levels. Here a score division of 0-30-80-100 (in percentage) for indicating the novice-intermediate-expert levels of knowledge integration is proposed (hypothesized) and summarized in Table III. Note that the identification of the score division is a process of posterior analysis, i.e., the assessment data is compared to the expected student behaviors predicted based on the knowledge integration levels defined in part 1 . Then, the score division, which can fit with a good agreement between test performance and expected problem-solving behaviors given in Tables I and II, is identified as the categorization scheme to match between total score and knowledge integration levels. However, since no additional measure of knowledge integration is conducted to independently categorize the knowledge integration levels, and the assessment outcomes are also population dependent, this score division scheme reflects only a reasonable approximation and should not be generally extended to other contexts and populations. Nevertheless, this work demonstrates the possibility for identifying a quantitative categorization scheme to model knowledge integration as well as its utility in teaching and learning.

Matching average scores to knowledge integration levels provides a convenient analysis rubric that can be easily used by teachers and researchers. In addition, the differences between the scores on different question sets can provide valuable information on the behaviors of students at all three levels of knowledge integration. To solve the typical questions in the single-link set, students only need to apply simple individual knowledge fragments. In traditional instruction, teachers often emphasize the result that "wave speed is related to the medium" with little explanation of the underlying mechanisms that cause the wave speed to be dependent only on the medium. This leads to students often relying on memorizing these results with little mechanistic understanding, and thus can only answer the familiar typical questions correctly.

The multilink set also includes typical questions but requires the activation of two or more linked knowledge components and correctly applying them in problem solving. As shown in Table III and Fig. 3, the mean score of the multilink set is significantly lower than that of the single-link set, indicating that most students' knowledge structures consist of individual knowledge elements with weak and local connections. As a result, they will have more trouble in solving questions that require multiple connected knowledge elements.

The integrated-link set contains atypical problems with contexts that are rarely seen in textbooks and lectures. In order to solve these questions correctly, students need to have a fully connected knowledge structure integrated

TABLE III. Summary of total score and question set scores per knowledge integration level based on combined pre- and post-test data. Standard errors are given in brackets.

\begin{tabular}{lccccc}
\hline \hline Knowledge integration level & Total score & $\mathrm{N}$ & Single-link typical & Multilink typical & Integrated-link atypical \\
\hline Novice & $0.0-30.0$ & 121 & $50.25(1.26)$ & $27.94(0.80)$ & $14.57(0.83)$ \\
Intermediate & $30.1-80.0$ & 408 & $73.85(0.67)$ & $48.17(0.88)$ & $28.90(0.67)$ \\
Expert & $80.1-100.0$ & 12 & $94.11(2.24)$ & $87.50(2.77)$ & $78.24(3.12)$ \\
\hline \hline
\end{tabular}


around the expert central idea of wave propagation. As can be seen from the result in Table III, only a small number of students were able to demonstrate this level of understanding. Most students used the particle-based FEM concept to solve these questions. For instance, a further inspection of common incorrect answers in Q3 shows that more than $67 \%$ of students responded that wave speed could be increased by increasing the frequency or the amplitude of the wave by shaking the string more rapidly. Similar results were also observed in the think-out-loud interviews discussed in part 1 of this research.

The results from part 2 demonstrate that the assessment design using different link-structure and typical and atypical contexts can be a useful strategy to assess knowledge integration. The assessment results have shown that the majority of students were in the intermediate level of knowledge integration, where they had only achieved a basic level performance on the multi-link typical questions but missed most of the integrate-link atypical questions. The fact that most students performed poorly on the questions that require more than a single link is a clear indication that the fragmentation of knowledge structure and lack of understanding of the expert central idea is quite prevalent among many students. This is likely due to the fact that traditional instruction often focuses on specific operational rules in problem solving, which only contributes to the development of single-link connections within students' knowledge structures but largely misses the expert central idea. In response to this issue, it is critical to develop new instructional strategies that explicitly emphasize the expert central idea to help students develop more integrated knowledge structures.

\section{PART 3: DESIGN AND EVALUATION OF A CONCEPTUAL-FRAMEWORK-BASED INSTRUCTION}

\section{A. Design of the instructional intervention}

Traditional physics instruction and problem-solving practices often emphasize routine strategies and operations to manipulate equations, without requiring comprehensive reasoning that uses the expert central idea. In such problemsolving training, only individual parts of the knowledge structure are activated at a time, although it is often expected that students would develop the necessary connections among the knowledge pieces through repeated exercises. However, as can be seen in the results from parts 1 and 2 of this research, a large number of students have failed to achieve a deep conceptual understanding of wave propagation after traditional instruction in high school physics courses. As a result, in part 3 of this research, a conceptual-framework-based instruction is developed that has a focus on helping students understand the expert central idea of wave propagation and apply it in various question contexts. It starts by explicitly emphasizing the development of the expert central idea in teaching. Then the instructor guides the students through problem-solving practices specifically designed to facilitate making connections between the central idea and other elements of the knowledge structure including different contextual variables and operational processes. Details of the teaching intervention are provided in the Supplemental Materials [55].

In the implementation of the teaching intervention, students were first asked to draw their own versions of the conceptual framework of wave propagation in groups of four, and then one student from each group was selected to explain the group's conceptual framework. Through this discussion of the conceptual framework, teachers were able to identify many common misconceptions, such as "the amplitude is the reflection of energy, and the elasticity is related to the source of wave"; "The wave source influences the frequency, period, and wavelength"; "The wave speed is related to both the wave source and the medium...The higher the oscillation frequency of the wave source is, the greater the wave speed is." Although all students have studied the topic of mechanical waves in their high school physic course and have practiced textbook problems extensively to prepare for the college admission exam, a large number of misconceptions still exist among these students, which are again demonstrated to be difficult to change through traditional instruction.

After students explained their conceptual frameworks, teachers then presented to the entire class the conceptual framework of wave propagation established in part 1. By doing so, students were explicitly exposed to both the expert and the novice central ideas, as well as a wide variety of reasoning pathways that a student may develop. Through reflective discussions and presentations, they could see the variety of understandings regarding different aspects of wave propagation from a more integrated perspective without being quickly settled into specific operations in problemsolving tasks like what they did in the traditional instruction.

After the introduction of the expert conceptual framework, instructors conducted an in-class activity with a group of 12 students in front of the whole class. In this activity, students form a single line to produce a moving wave by having successive students standing up, raising their arms, lowering their arms, and sitting down again. This activity allows students to simulate being the "medium" of a cross-sectional mechanical wave and experienced how such a wave propagates through the medium, which provides an explicit and engaging real-world setting for applying the expert central idea. Following the hands-on activities, problem-solving examples were also introduced and practiced in class, which was designed to further reinforce students' understanding of the expert central idea and practice its application in different problem-solving settings.

The design of the conceptual-framework-based instruction provides students multiple opportunities to investigate and reflect on various applications of the expert central idea 


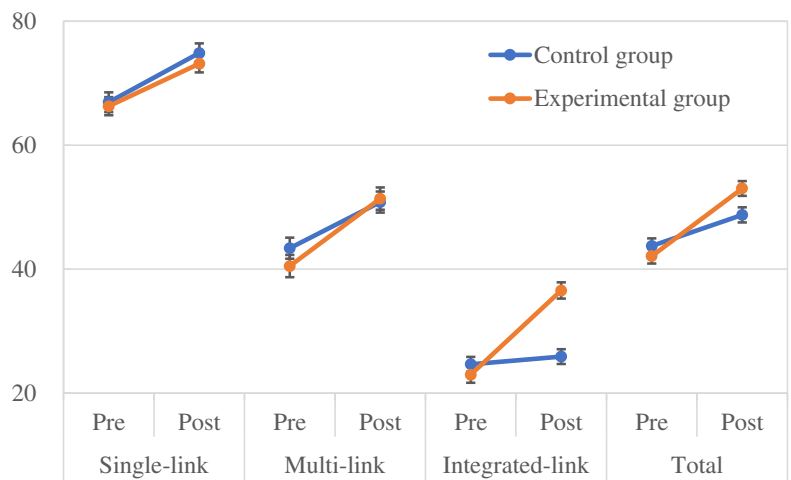

FIG. 4. Pretest and post-test performances of students in the control and experimental groups for three question sets. The error bars represent the standard errors of the means.

and to extend their capacity of applying the expert central idea in complex problem settings. With the explicit emphasis on teaching the expert central idea and practicing its application in problem solving, it is expected that students will be able to develop a more integrated knowledge structure and achieve a deeper conceptual understanding of wave propagation.

\section{B. Implementation and evaluation of the instructional intervention}

The main goal of this study is to implement the new teaching intervention and evaluate its effectiveness compared to traditional instruction. Out of the 312 students who took the pretest, 229 students in 5 classes were selected into two groups, a control group with 121 students in 3 classes and an experimental group with 108 students in 2 classes. The students in the control group received the traditional instruction taught by one instructor, while the students in the experimental group received the new instruction taught by another instructor. Both instructors have a comparable background with approximately 7 years of experience teaching this course.

The format of instruction is also controlled, such that both groups used the same in-class activities and received similar problem-solving practices, i.e., the example problems given during lectures and the homework assignments were identical for the two groups. The main difference is that the new instruction makes explicit emphasis on introducing and practicing the expert central idea in group activities and problem-solving practices, which is expected to help students make more integrated connections between the central idea and other elements of their knowledge. Meanwhile, the traditional instruction followed the traditional curriculum with the same format of teaching but lacked the explicit emphasis on the expert central idea.

Both groups took the post-test immediately after they had finished learning the mechanical waves. Statistical significance of the difference between the two groups was evaluated with three-way ANOVA, and further explored through $t$ tests and Cohen's $d$ effect size. For the analysis in this study, the matched pre- and post-test data from the 229 students were used. The three-way ANOVA is a $2 \times 2 \times 3$ mixed design. The pre- and post-test performances of the 229 students across three question sets and two instruction methods are compared to determine the effectiveness of the new instruction method. The results are plotted in Fig. 4 and listed in Table IV. The results show a significant interaction among the instruction conditions (traditional and new instruction), test conditions (pre- and post-test), and question sets (single link, multilink, and integrated link) $[F(2,1362)=5.011, p=0.007]$.

For the pretest performance, the result of a two-way ANOVA reveals no interaction between instruction conditions and question sets $[F(2,681)=0.267, p=0.766]$, indicating that the two groups were from a single homogeneous population. On the other hand, the result of a two-way ANOVA of the post-test shows significant interactions between instruction conditions and question sets $[F(2,681)=9.420, p<0.001]$, with the main effect for three question sets $[F(2,681)=396.217, p<0.001]$ and instruction condition $[F(1,681)=6.520, p=0.011]$. As shown in Fig. 4, the pre- and post-test scores of the control and experimental groups on the single-link and multilink question sets are nearly identical $(p>0.412)$. However, the integrated-link set sees significant difference between the two groups $\left[t_{\text {Inte }}(227)=6.06, p<0.001, d=0.81\right]$.

To further examine the impact of instruction on learning, pre-post score changes are compared based on the results in

TABLE IV. Summary of students' pre- and post-test performances.

\begin{tabular}{|c|c|c|c|c|c|c|c|c|}
\hline \multirow[b]{2}{*}{ Question set } & \multirow[b]{2}{*}{ Group } & \multicolumn{2}{|c|}{ Pretest } & \multicolumn{2}{|c|}{ Post-test } & \multirow[b]{2}{*}{$t$ value } & \multirow[b]{2}{*}{$p$ value } & \multirow[b]{2}{*}{$d$} \\
\hline & & Mean & SE & Mean & SE & & & \\
\hline \multirow[t]{2}{*}{ Single-link set (typical questions) } & Control & 66.94 & 1.59 & 74.88 & 1.55 & 6.70 & $<0.001$ & 0.46 \\
\hline & Experimental & 66.30 & 1.46 & 73.15 & 1.40 & 6.69 & $<0.001$ & 0.46 \\
\hline \multirow[t]{2}{*}{ Multilink set (typical questions) } & Control & 43.39 & 1.70 & 50.83 & 1.73 & 5.87 & $<0.001$ & 0.39 \\
\hline & Experimental & 40.51 & 1.64 & 51.39 & 1.83 & 7.96 & $<0.001$ & 0.60 \\
\hline \multirow[t]{2}{*}{ Integrated-link set (atypical questions) } & Control & 24.66 & 1.52 & 25.90 & 1.19 & 1.32 & 0.191 & 0.08 \\
\hline & Experimental & 22.99 & 1.11 & 36.57 & 1.31 & 11.47 & $<0.001$ & 1.08 \\
\hline \multirow[t]{2}{*}{ Total } & Control & 43.75 & 1.14 & 48.76 & 1.19 & 6.22 & $<0.001$ & 0.39 \\
\hline & Experimental & 42.10 & 0.98 & 53.02 & 1.22 & 10.91 & $<0.001$ & 0.95 \\
\hline
\end{tabular}


Table IV, which show significant pre-post gains on the total score for both the control and the experimental groups. For the individual question sets, the pre-post gains are significant in all conditions except for the control group on the integrated-link set. Comparing between the experimental and control groups, both groups achieved similar gains on single-link and multilink questions $(p>0.066)$. Meanwhile, the experimental group achieved a significantly larger gain on the integrated-link set than the control group did $(p<0.001)$.

The results suggest that both the traditional and new instruction methods are similarly effective in improving students' problem-solving performances on simple and complex typical questions. However, only the new instruction method, which emphasizes the development of the expert central idea, can significantly improve students' performances on the atypical questions. The results indicate that the emphasis on the expert central idea in instruction is effective in helping students develop integrated links in their knowledge structures and gain a deeper conceptual understanding of wave propagation.

\section{CONCLUSION}

In this research, a conceptual framework of wave propagation is established to guide the assessment and instruction of students' knowledge integration in understanding wave propagation. Based on the analysis of interviews and assessment data, students' understanding is categorized into three developmental levels of knowledge integration including novice, intermediate, and expert.

Students in the novice level held the novice central idea consistently, directly connecting the contextual features and variables to the particle-based concepts. In problem solving, these students mostly used force, energy, or kinematic motion concepts to explain their answers to wave propagation problems, which are consistent with results demonstrated in previous studies on wave propagation $[38,43,45]$. Students at this level were able to correctly solve some simple typical questions using memorized operations or equations but often failed on more complex typical questions and atypical questions.

Students in the intermediate level began to develop multiple local links that include elements from both the expert model and the novice model. However, these local links in students' knowledge structures were not connected to the expert central idea, which often led to inconsistencies in their reasoning. As a result, in problem solving, students often applied parts of the novice and expert models inconsistently in different question contexts. The context sensitivity of the activation of knowledge pieces has been extensively studied and appears to be a signifying feature of students in the intermediate transitional stage of learning [20-22,34,41]. Students at this level often exhibit having multiple transitional thinking pathways in the process of problem solving. The use of conceptual framework model can help represent such multifaceted thinking pathways and provide an operational modeling framework for guiding assessment. Based on the assessment outcomes of this research, students at this level were found to be able to correctly solve the single-link and some multilink questions but usually fail on the atypical questions.

Students in the expert level achieved a more integrated knowledge structure and a deeper conceptual understanding of wave propagation, which allowed them to apply the expert central idea consistently in different contexts. In terms of their knowledge structures, the expert central idea acted as an anchor node that robustly connects all components of the conceptual framework to form an integrated network of knowledge that can be successfully applied in familiar and novel situations. Students at this level were able to correctly solve all types of typical questions as well as most atypical questions.

Building off the conceptual framework and assessment outcomes, a new teaching intervention was designed with an emphasis on helping students develop a good understanding of the expert central idea. A controlled study was conducted to evaluate the effectiveness of the teaching intervention, which showed that both the traditional instruction and the new instruction had a similar impact on students' pre-post score gains on typical questions but only the new instruction made significant improvement on the atypical questions. The results suggest that emphasizing the understanding and application of the expert central idea during instruction can help students develop more integrated knowledge structures and gain a deeper conceptual understanding of wave propagation.

Although encouraging outcomes have been observed, there are a few limitations to this research, which should be further examined in future studies. First, the population studied in this research has only a small number of advanced students, which limits the scope of the analysis on higher intermediate and expert level students. The intermediate level students appear to have a wide range of reasoning pathways. It will be valuable to further investigate finer subcategories within the broad intermediate level currently defined, which is not feasible in this study due to the limited number of more advanced students. It is then beneficial to study a population with a large number of advanced students so that a more complete developmental progression of knowledge integration on wave propagation can be examined.

Another limitation is that the teaching intervention is only in its early stage of development with limited success. It will be useful to further improve the intervention to enhance its effectiveness and test its impact on different student populations. All the limitations of the current study should certainly warrant future research to further improve instruction on student learning.

In conclusion, this paper documents a new application of the conceptual framework model to the concept of wave propagation and introduces a dual-structure conceptual 
framework that explicitly shows the expert model as well as the novice model. Guided by the conceptual framework, an assessment and a teaching intervention were developed and tested. The results provide encouraging evidence suggesting that the conceptual framework model can be a valuable guide to the development of assessment and instruction for promoting knowledge integration and deep learning.

\section{ACKNOWLEDGMENTS}

This work is supported in part by the NSF Grant No. DUE-1712238, as well as Humanities and Social Sciences Project of the Ministry of Education of China (No. 18YJC880094), Teaching Reform Project in Higher Education of Hubei Province in P.R.C. (No. 2018288, No. 2018295).
[1] P. G. Hewitt, Millikan Lecture 1982: The missing essential - a conceptual understanding of physics, Am. J. Phys. 51, 305 (1983).

[2] D. Huffman and P. Heller, What does the Force Concept Inventory actually measure?, Phys. Teach. 33, 138 (1995).

[3] P. V. Engelhardt and R. J. Beichner, Students' understanding of direct current resistive electrical circuits, Am. J. Phys. 72, 98 (2004).

[4] E. Gaigher, J. M. Rogan, and M. W. H. Braun, Exploring the development of conceptual understanding through structured problem solving in physics, Int. J. Sci. Educ. 29, 1089 (2007).

[5] P. Nieminen, A. Savinainen, and J. Viiri, Relations between representational consistency, conceptual understanding of the force concept, and scientific reasoning, Phys. Rev. ST Phys. Educ. Res. 8, 010123 (2012).

[6] L. C. McDermott and E. F. Redish, Resource letter: PER-1: Physics education research, Am. J. Phys. 67, 755 (1999).

[7] A. E. Rivet and J. S. Krajcik, Contextualizing instruction: Leveraging students' prior knowledge and experiences to foster understanding of middle school science, J. Res. Sci. Teach. 45, 79 (2008).

[8] A. Elby, Another reason that physics students learn by rote, Am. J. Phys. 67, S52 (1999).

[9] D. R. Krathwohl, A revision of Bloom's taxonomy: An overview, Theory Into Practice 41, 212 (2002).

[10] NGSS Lead States, Next Generation Science Standards: For States, by States (National Academies Press, Washington, DC, 2013).

[11] M. C. Linn, The knowledge integration perspective on learning and instruction, in The Cambridge Handbook of the Learning Sciences, edited by K. Sawyer (Cambridge University Press, New York, 2006), pp. 243-264.

[12] L. Bao and K. Koenig, Physics education research for 21st century learning, Discip. Interdiscip. Sci. Educ. Res. 1, 1 (2019).

[13] A. A. DiSessa, Toward an epistemology of physics, Cognit. Instr. 10, 105 (1993).

[14] A. A. Disessa and B. L. Sherin, What changes in conceptual change?, Int. J. Sci. Educ. 20, 1155 (1998).

[15] G. Posner, K. Strike, P. Hewson, and W. Gertzog, Accommodation of a scientific conception: Toward a theory of conceptual change, Sci. Educ. 66, 211 (1982).

[16] S. Vosniadou, Capturing and modeling the process of conceptual change, Learning Instr. 4, 45 (1994).
[17] M. T. Chi, J. D. Slotta, and N. De Leeuw, From things to processes: A theory of conceptual change for learning science concepts, Learning Instr. 4, 27 (1994).

[18] M. T. Chi, Commonsense conceptions of emergent processes: Why some misconceptions are robust, J. Learn. Sci. 14, 161 (2005).

[19] A. Gupta, D. Hammer, and E. F. Redish, The case for dynamic models of learners' ontologies in physics, J. Learn. Sci. 19, 285 (2010).

[20] D. Hammer, Student resources for learning introductory physics, Am. J. Phys. 68, S52 (2000).

[21] M.S. Sabella and E. F. Redish, Knowledge organization and activation in physics problem solving, Am. J. Phys. 75, 1017 (2007).

[22] L. M. Goodhew, A. D. Robertson, P. R. L. Heron, and R. E. Scherr, Student conceptual resources for understanding mechanical wave propagation, Phys. Rev. Phys. Educ. Res. 15, 020127 (2019).

[23] O. L. Liu, H. S. Lee, and M. C. Linn, Measuring knowledge integration: Validation of four-year assessments, J. Res. Sci. Teach. 48, 1079 (2011).

[24] M. C. Linn, H. S. Lee, R. Tinker, F. Husic, and J. L. Chiu, Teaching and assessing knowledge integration in science, Science 313, 1049 (2006).

[25] R. Dai, J. C. Fritchman, Q. Liu, Y. Xiao, H. Yu, and L. Bao, Assessment of student understanding on light interference, Phys. Rev. Phys. Educ. Res. 15, 0201342019.

[26] Y. Nie, Y. Xiao, J. C. Fritchman, Q. Liu, J. Han, J. Xiong, and L. Bao, Teaching towards knowledge integration in learning force and motion, Int. J. Sci. Educ. 41, 2271 (2019).

[27] W. Xu, Q. Liu, K. Koenig, J. Fritchman, J. Han, S. Pan, and L. Bao, Assessment of knowledge integration in student learning of momentum, Phys. Rev. Phys. Educ. Res. 16, 010130 (2020).

[28] L. Bao and J. C. Fritchman, Knowledge integration in student learning of Newton's third law: Addressing the action-reaction language and the implied causality, Phys. Rev. Phys. Educ. Res. 17, 020116 (2021).

[29] J. Larkin, J. McDermott, D. P. Simon, and H. A. Simon, Expert and novice performance in solving physics problems, Science 208, 1335 (1980).

[30] M. T. Chi, P. J. Feltovich, and R. Glaser, Categorization and representation of physics problems by experts and novices, Cogn. Sci. 5, 121 (1981). 
[31] D. Hestenes, Toward a modeling theory of physics instruction, Am. J. Phys. 55, 440 (1987).

[32] P. T. Hardiman, R. Dufresne, and J. P. Mestre, The relation between problem categorization and problem solving among experts and novices, Memory Cognit. 17, 627 (1989).

[33] J. L. Snyder, An investigation of the knowledge structures of experts, intermediates and novices in physics, Int. J. Sci. Educ. 22, 979 (2000).

[34] L. Bao and E. F. Redish, Model analysis: Representing and assessing the dynamics of student learning, Phys. Rev. ST Phys. Educ. Res. 2, 010103 (2006).

[35] L. Bao, K. Hogg, and D. Zollman, Model analysis of fine structures of student models: An example with Newton's third law, Am. J. Phys. 70, 766 (2002).

[36] N. B. Songer and M. C. Linn, How do students' views of science influence knowledge integration?, J. Res. Sci. Teach. 28, 761 (1991).

[37] R. Duit and D. F. Treagust, Conceptual change: A powerful framework for improving science teaching and learning, Int. J. Sci. Educ. 25, 671 (2003).

[38] M. Wittmann, R. N. Steinberg, and E. F. Redish, Understanding and affecting student reasoning about sound waves, Int. J. Sci. Educ. 25, 991 (2003).

[39] A. Tongchai, M. D. Sharma, I. D. Johnston, K. Arayathanitkul, and C. Soankwan, Developing, evaluating and demonstrating the use of a conceptual survey in mechanical waves, Int. J. Sci. Educ. 31, 2437 (2009).

[40] P. Barniol and G. Zavala, Mechanical waves conceptual survey: Its modification and conversion to a standard multiple-choice test, Phys. Rev. ST Phys. Educ. Res. 12, 010107 (2016).

[41] A. Tongchai, M. D. Sharma, I. D. Johnston, K. Arayathanitkul, and C. Soankwan, Consistency of students' conceptions of wave propagation: Findings from a conceptual survey in mechanical waves, Phys. Rev. ST Phys. Educ. Res. 7, 020101 (2011).

[42] L. Maurines, Spontaneous reasoning on the propagation of visible mechanical signals, Int. J. Sci. Educ. 14, 279 (1992).

[43] M. C. Wittmann, R. N. Steinberg, and E. F. Redish, Making sense of how students make sense of mechanical waves, Phys. Teach. 37, 15 (1999).

[44] C. J. Linder, University physics students' conceptualizations of factors affecting the speed of sound propagation, Int. J. Sci. Educ. 15, 655 (1993).

[45] M. C. Wittmann, The object coordination class applied to wave pulses: Analysing student reasoning in wave physics, Int. J. Sci. Educ. 24, 97 (2002).
[46] I. S. Caleon and R. Subramaniam, Exploring students' conceptualization of the propagation of periodic waves, Phys. Teach. 48, 55 (2010).

[47] A. Pejuan, X. Bohigas, X. Jaén, and C. Periago, Misconceptions about sound among engineering students, J. Sci. Educ. Technol. 21, 669 (2012).

[48] M. Kryjevskaia, M. R. Stetzer, and P. R. Heron, Student understanding of wave behavior at a boundary: The relationships among wavelength, propagation speed, and frequency, Am. J. Phys. 80, 339 (2012).

[49] P. Barniol and G. Zavala, The mechanical waves conceptual survey: An analysis of university students' performance, and recommendations for instruction, Eurasia J. Math. Sci. Technol. Educ. 13, 929 (2017).

[50] E. E. Clough and R. Driver, A study of consistency in the use of students' conceptual frameworks across different task contexts, Sci. Educ. 70, 473 (1986).

[51] D. Palmer, How consistently do students use their alternative conceptions?, Res. Sci. Educ. 23, 228 (1993).

[52] Z. Hrepic, D. A. Zollman, and N. S. Rebello, Identifying students' mental models of sound propagation: The role of conceptual blending in understanding conceptual change, Phys. Rev. ST Phys. Educ. Res. 6, 020114 (2010).

[53] D. E. Brown, Students' conceptions as dynamically emergent structures, Sci. Educ. 23, 1463 (2014).

[54] T. R. Thoads and R. J. Roedel, The wave concept inventory-a cognitive instrument based on Bloom's taxonomy, in Proceedings of the FIE'99 Frontiers in Education. 29th Annual Frontiers in Education Conference. Designing the Future of Science and Engineering Education (IEEE, San Juan, PR, USA, 1999).

[55] See Supplemental Material at http://link.aps.org/ supplemental/10.1103/PhysRevPhysEducRes.17.020122, for details of the Concept Test of Mechanical Wave Propagation and a conceptual-framework-based intervention lesson plan.

[56] J. L. Chiu and M. C. Linn, Supporting knowledge integration in chemistry with a visualization-enhanced inquiry unit, J. Sci. Educ. Technol. 23, 37 (2014).

[57] J. Biggs and K. Collis, Evaluating the Quality of Learning: The SOLO taxonomy (Academic Press, New York, 1982).

[58] R.F. DeVellis, Scale Development: Theory and Applications (Sage Publications, Thousand Oaks, CA, 2012), Vol. 26. 